\title{
Correction to: Composite Post Insulators
}

\section{Correction to: Chapter 3 in:}

K. O. Papailiou and F. Schmuck, Silicone Composite Insulators, Power Systems, https://doi.org/10.1007/978-3-642-15320-4_3

The original version of this chapter was published with incorrect function in the equation as "tan", which is now corrected to "tanh". The original chapter has been corrected. 